\title{
New Pharmacist Role in Diabetes Education in Sri Lanka: A Cross- Sectional Descriptive Randomized Step-Up Study
}

\section{Bulathsinghalage Poornima Reshamie Cooray ${ }^{1}$, Hana Morrissey ${ }^{2 *}$, Eisha Indumani Waidyarathne ${ }^{3}$, Patrick Anthony Ball ${ }^{2}$ and Manilka Sumanathilake}

\author{
${ }^{1}$ Department of Pharmacy, University of Ruhuna, Sri Lanka \\ ${ }^{2}$ School of Pharmacy, University of Wolverhampton, United Kingdom \\ ${ }^{3}$ Medical Faculty, University of Ruhuna, Sri Lanka \\ ${ }^{4}$ Diabetes and Endocrinology Unit, Teaching Hospital Karapitiya, Sri Lanka
}

*Corresponding author: Dr. Hana Morrissey, Faculty of Science and Engineering, School of Pharmacy, University of Wolverhampton, Wulfruna Street, Wolverhampton, WV1 1LY, United Kingdom, Tel: +44-(0)-1902-322173, E-mail:

Hana.Morrissey@wlv.ac.uk

\begin{abstract}
Introduction: The prevalence of Type 2 diabetes is globally on the rise, in both developed and developing countries. Type 2 diabetes is a major public health issue in Sri Lanka. This study aims to investigate the effect of structured self-management health education intervention based on 'PITS model' (Pathophysiology, Indications, Treatment and Specifics) would result in a clinically significant improvement in glycaemic control of type 2 Diabetes Mellitus (T2DM) patients.

Methods: Patients who were diagnosed with T2DM at two tertiary care hospitals in Sri Lanka, comply with the selection criteria were enrolled to the study. The intervention consisted with two repeated one to one education sessions followed up in six and twelve months. $\mathrm{HbA1c}$, lipid profiles, waist circumference, BMI and other biomedical measurements were done in both groups. Analysis of covariance between groups were conducted to determine the effectiveness of the intervention.
\end{abstract}

Results: Mean $\mathrm{HbA} 1 \mathrm{c}$ level in both intervention and usual care group was $8.6 \%$ with deviation from their target glycaemic level $(6.5 \%, 48 \mathrm{mmol} / \mathrm{mol})$ at baseline. At six months, there was a significant reduction $(P<0.001$; size of effect $=$ 0.69 ) in $\mathrm{HbA} 1 \mathrm{c}$ between the intervention and the usual care group controlling the baseline values.

Conclusion: The results demonstrate the effectiveness of one to one diabetes self-management intervention among the adults with T2DM.

\section{Key words}

Diabetes self-management, Type 2 Diabetes, One to one patient education, $\mathrm{HbA} 1 \mathrm{c}$ level

\section{Introduction}

Diabetes mellitus is a non-communicable disease, affecting more than 180 million people worldwide. It is estimated to reach 592 million by 2035; of which the majority are diagnosed with type 2 diabetes (T2DM) [1-3]. Sri Lanka is one of the countries with the highest diabetes prevalence rates in the world. There were 1.16 million cases of all types of diabetes were recorded in Sri Lanka in 2016 as stated in an International Federation of Diabetes Atlas [4].

Complications of T2DM due to poor control lead to early disability which is not only debilitating to those affected, but also a burden on their communities, healthcare services and the socioeconomic status of the country [4-6]. Diabetes self-management plays a vital role in management of diabetes and the diabetes education on lifestyle modifications is the major contributor in optimising the metabolic control of $\operatorname{T2DM}[7,8]$.

In many developing countries, especially in South Asia, primary care for T2DM patients is provided through local government-funded hospitals that are usually overcrowded with limited patient-physician interactions, which 
may have an indirect impact on poor diseases prognosis and which in-turn increase the burden of health expenses due to complications $[9,10]$. Thus, the attitude of the healthcare team towards time spent in patient education and the quality of such education is not clear or structured, and its effect on the patient glycaemic control is not clearly measured. Recent evidence of studies from Perera, et al. and Jayawardena, et al. reported the limited patients' knowledge on the management of their disease in Sri Lanka which underlines an urgent need of setting an effective culturally sensitive and appropriate education program on lifestyle modification. However, 'Chronic care models have extensively practiced for self-management programs, but to our knowledge, no patient education studies based on 'PITS model' (Pathophysiology, Indications, Treatment and Specifics) has been practiced in Sri Lanka [10-12]. The PITS model is a logical, organised universal teaching model that presents information which enhances the receiver's ability to recall information $[13,14]$.

Therefore, this study was designed with the aim to examine the impact of a culturally appropriate, health-education on life style modification and self-management on patient diagnosed with diabetes to improve their glycaemic control and delay disease complications.

This paper provides an overview of baseline characteristics on enrolment and the significance of an intervention after six months.

\section{Methods}

\section{Study design and selection of samples}

This study was designed as a cross sectional descriptive randomised step up study of a one-on-one health education as the main intervention. It was conducted in two main tertiary care facilities in Western and Southern Provinces of Sri Lanka; where the prevalence of diabetes is highest and third highest respectively [15]. The sample size was calculated based on the ability to detect a statistically significant difference in HBA1c level and a convenience sample size. A total of 150 participants was considered as a manageable sample that would provide sufficient data to inform future studies on the trends, variances and relationships, between self-care behaviour, level of health education and diabetes control. The estimated withdrawal rate for the study was expected to be approximately $10 \%[4,16]$.

Patients who were diagnosed with type 2 diabetes; all genders above age of 18 years at the time of recruitment were invited to enrol. Participants who were pregnant, with gestational diabetes or Type 1 diabetes and those who were on haemodialysis, unable to speak or understand Sinhala or those identified as having cognitive, hearing or vision impairment were excluded from the study.

The study participants were selected based on every third patient from the clinic registry in both 'usual care' and intervention groups at the beginning of the study. A computer-generated algorithm using Microsoft Excel ${ }^{\mathrm{TM}}$ was adapted to further randomise the participants into subgroups within the intervention groups to determine the effect of the education session among subgroups.

\section{Ethical consideration}

Ethical approval was obtained from Human Research Ethics Committee (HREC), Charles Darwin University (Ref. No: HI4082), Australia and Ethics Review Committee, Faculty of Medicine (Ref No: 17.11.2014:3.32), University of Ruhuna, Sri Lanka.

The site approval was obtained from Directors of both tertiary care facilities in Sri Lanka. Potential participants were informed verbally and in writing about the study objectives and processes, their rights, privacy of the participants been maintained and what was expected of them if they decided to participate. Written, informed consent was obtained in Sinhala, their native language and their participation was on a voluntary basis.

\section{Study tools and data collection}

The intervention and non-intervention participants, who continued receiving their usual diabetes care, were recruited from two main independent tertiary care facilities in Western and Southern Provinces of Sri Lanka.

The assignment of participants was carried out based on computer generated random allocation for the intervention subgroups and non-intervention group, enrolment of participants and assignment of participants to the study was carried out by the primary investigator.

The research method was based on two main aspects the clinical data collection and the diabetes health-education intervention. Clinical data collection took place on three occasions for all intervention and non-intervention participants - at the enrolment day, six-month follow up and 12-month follow up, while health education was carried out among the intervention group in three occasions. Participants were blinded throughout the study by without informing that they receive single, repeated or no education intervention and assess their improvement of knowledge on diabetes.

The participants were provided with two questionnaires by the main investigator. The first one is a researcher administered questionnaire that included basic demographic data, the level of education, medical and medication history, lifestyle and their anthropometric measurements. The second questionnaire was a structured health education questionnaire (health education impact questionnaire -heiQ ${ }^{\mathrm{TM}}$ ), translated (back and forward translation) into Sinhala language to measure the participants' knowledge and attitude towards their self-care on pre-and post-health education session. This is the first time the HeiQ ${ }^{\text {TM }}$ was used in Sinhala language. The $\mathrm{HeiQ}^{\mathrm{TM}}$ was used under the licensed agreement with the Deakin University, Australia where 
it was developed, translated and validated for meaning and cognitive interpretation through professional translators $[17,18]$.

Participant ID was developed to maintain consistency between the non-intervention (THKC) and the intervention groups (THKA or THKB) and to avoid mixing of the data, thereby ensuring the data for each participant were precisely collated throughout the study.

Participants from the intervention group received computer based Excel randomised identification (ID) serial numbers to either Subgroup A or B; 50 participants for each group. However, in anticipation of a $10 \%$ dropout rate, 55 participants were enrolled to each group. Participants were allocated with a study ID number regardless of being in Subgroup A or B. 50 participants were also allocated for non-intervention group.

The completion of enrolment was performed by participants signing the Sinhala translated written consent form. The enrolment of participants was completed within the first two months of the study and no replacement of participants during the study applied. The number of participants approached by the researcher who immediately declined to participate or withdrew from the study during the intervention period were also recorded and no changes to the methods after trial commencement occurred.

This research did not interfere with the patients' current prescribed medications or the individuals' diet. There was no administering of any medications or testing any therapeutic regimen. However, patients were referred to their treating doctor if any risk was identified. Data were collected by the principal investigator herself and phlebotomists were recruited to collect blood samples for $\mathrm{HbA} 1 \mathrm{c}$ and Lipid profile tests. The phlebotomists were trained to comply with the site infection control and blood handling. A $5 \mathrm{ml}$ venous blood samples were collected from the median cubital vein, cephalic vein or the basilic vein in the antecubital site or from the basilic, cephalic or dorsal metacarpal veins in the wrist. Gold standard was adopted to determine the level of $\mathrm{HbA1C}$ and all tests (Lipid profile and $\mathrm{HbA} 1 \mathrm{c}$ tests) were performed at an accredited ISO 9001 certified laboratory.

Followed by the completion of questionnaires, the intervention group received the structured health education program on enrolment in one-to-one basis by the principal investigator. The program comprised of a multimedia presentation that includes the pathogenesis, progression and complications of T2DM; importance of proper management and follow-up. Also, it demonstrated the use of blood glucose monitors and insulin pens. The session then progressed to focus on the participant prescribed medication mode of action, side effects and the effect of adherence to medication on disease prognosis and development of complications. The 'usual care' group from the second hospital received the same questionnaires as the intervention group without the health education session.

These scheduled one-to-one structured education sessions contribute to the teaching interaction between participant and educator, enabling participant control.

The main outcome of the study recognised as primary outcome with measurement of an improvement in participant $\mathrm{HbA} 1 \mathrm{c}$ and improving their condition,

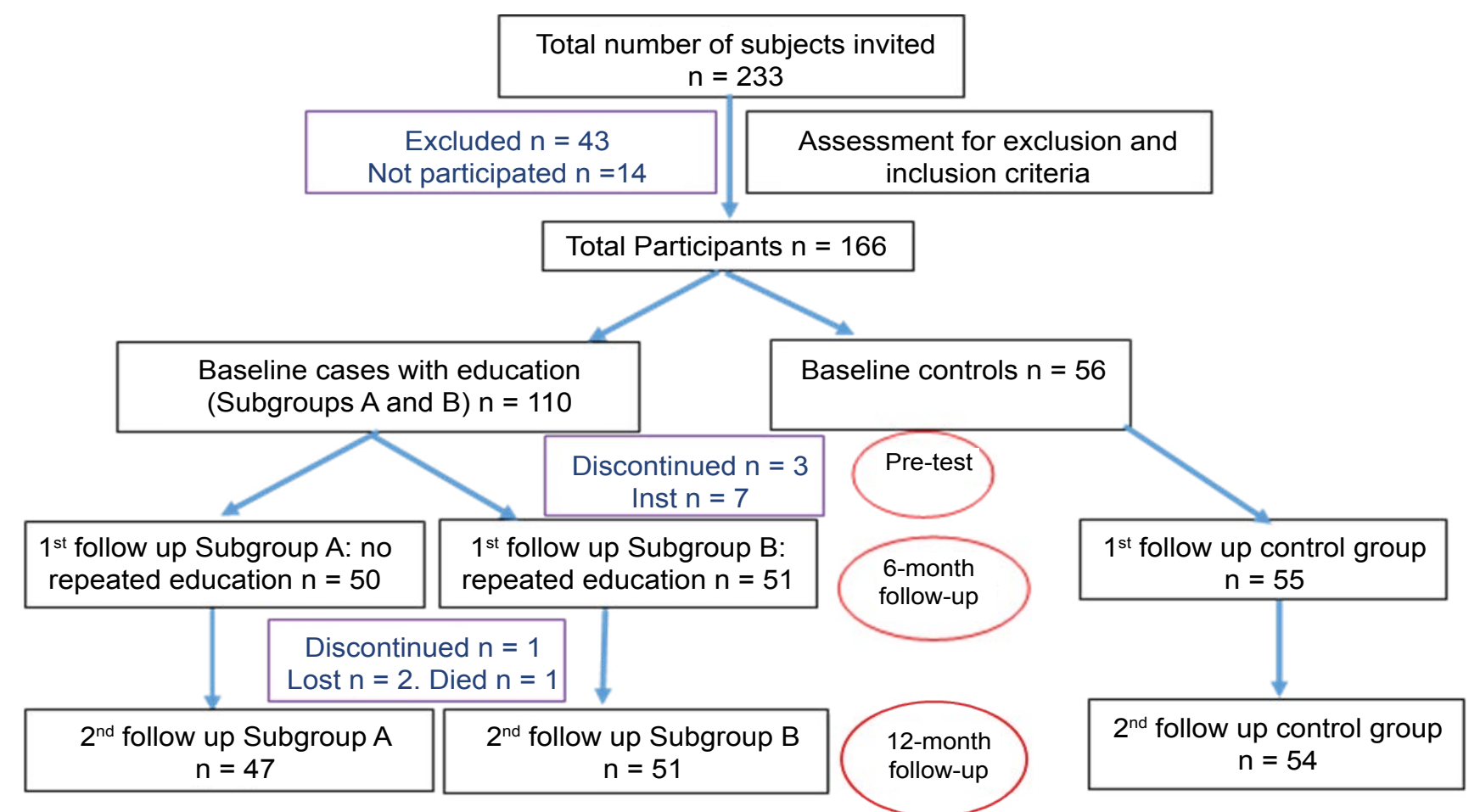

Figure 1: Consort diagram. 
Table 1: Summary Statistics for usual care and intervention groups at baseline and six months.

\begin{tabular}{|c|c|c|c|c|c|c|c|c|c|c|c|}
\hline \multirow{3}{*}{ Measurement } & \multirow{3}{*}{ Time } & \multirow{2}{*}{\multicolumn{5}{|c|}{$\begin{array}{l}\text { Intervention } \\
(\mathbf{n}=\mathbf{1 1 0}) \\
\text { Usual Care }(G p C)\end{array}$}} & \multirow{2}{*}{\multicolumn{3}{|c|}{$\begin{array}{l}\text { Non-intervention } \\
(\mathbf{n}=\mathbf{5 6})\end{array}$}} & \multicolumn{2}{|c|}{$\begin{array}{l}\text { Total } \\
(\mathrm{n}=166)\end{array}$} \\
\hline & & & & & & & & & & & \\
\hline & & $z$ & $\begin{array}{l}\frac{c}{\mathbb{d}} \\
\mathbb{\mathbb { d }} \\
\sum\end{array}$ & 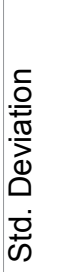 & 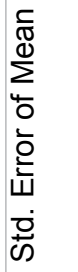 & 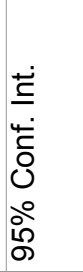 & $z$ & $\begin{array}{l}\frac{c}{\sqrt{\mathbb{N}}} \\
\stackrel{\mathbb{d}}{\Sigma}\end{array}$ & $\begin{array}{l}\frac{}{0} \\
\frac{\pi}{2} \\
\frac{\pi}{0} \\
\dot{0} \\
\dot{0}\end{array}$ & 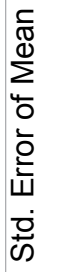 & 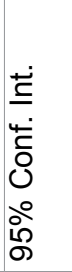 \\
\hline \multirow[t]{2}{*}{ HbA1c level (\%) } & baseline & 56 & 8.65 & 1.45 & 0.19 & 0.39 & 110 & 8.56 & 1.62 & 0.15 & 0.31 \\
\hline & 6 months & 55 & 8.23 & 1.30 & 0.17 & 0.35 & 101 & 7.51 & 1.12 & 0.11 & 0.22 \\
\hline \multirow[t]{2}{*}{ Systolic Pressure $(\mathrm{mmHg})$} & baseline & 56 & 129.4 & 16.7 & 2.2 & 4.5 & 110 & 126.3 & 16.2 & 1.5 & 3.1 \\
\hline & 6 months & 55 & 126.8 & 14.2 & 1.9 & 3.8 & 101 & 123.9 & 18.4 & 1.8 & 3.7 \\
\hline \multirow[t]{2}{*}{ Diastolic Pressure $(\mathrm{mmHg})$} & baseline & 56 & 84.9 & 9.1 & 1.2 & 2.4 & 110 & 79.6 & 9.4 & 0.9 & 1.8 \\
\hline & 6 months & 55 & 84.6 & 10.0 & 1.3 & 2.7 & 101 & 81.1 & 9.9 & 1.0 & 2.0 \\
\hline \multirow[t]{2}{*}{ Heart rate (bpm) } & baseline & 56 & 81.1 & 11.0 & 1.5 & 2.9 & 110 & 78.4 & 11.6 & 1.1 & 2.2 \\
\hline & 6 months & 55 & 75.3 & 10.2 & 1.4 & 2.8 & 101 & 77.5 & 15.6 & 1.6 & 3.1 \\
\hline \multirow[t]{2}{*}{ Total Cholesterol (mg/dL) } & baseline & 56 & 181.3 & 38.9 & 5.2 & 10.4 & 110 & 189.8 & 44.1 & 4.2 & 8.4 \\
\hline & 6 months & 55 & 179.7 & 38.0 & 5.1 & 10.3 & 101 & 173.8 & 35.7 & 3.6 & 7.1 \\
\hline \multirow[t]{2}{*}{ HDL Cholesterol (mg/dL) } & baseline & 56 & 45.0 & 9.0 & 1.2 & 2.4 & 110 & 45.1 & 8.5 & 0.8 & 1.6 \\
\hline & 6 months & 55 & 43.1 & 7.3 & 1.0 & 2.0 & 101 & 46.4 & 13.2 & 1.3 & 2.6 \\
\hline \multirow[t]{2}{*}{ Triglycerides (mg/dL) } & baseline & 56 & 129.1 & 46.7 & 6.2 & 12.5 & 110 & 126.3 & 64.4 & 6.1 & 12.3 \\
\hline & 6 months & 55 & 118.0 & 53.1 & 7.2 & 14.3 & 101 & 115.2 & 43.9 & 4.4 & 8.7 \\
\hline \multirow[t]{2}{*}{ LDL Cholesterol (mg/dL) } & baseline & 56 & 109.5 & 35.9 & 4.8 & 9.6 & 110 & 100.1 & 35.2 & 3.4 & 6.7 \\
\hline & 6 months & 55 & 107.9 & 34.2 & 4.6 & 9.2 & 101 & 107.7 & 31.2 & 3.1 & 6.2 \\
\hline \multirow[t]{2}{*}{ BMI $\left(\mathrm{kg} / \mathrm{m}^{2}\right)$} & baseline & 56 & 24.7 & 3.45 & 0.46 & 0.92 & 110 & 24.8 & 4.25 & 0.40 & 0.81 \\
\hline & 6 months & 55 & 24.9 & 3.70 & 0.50 & 1.00 & 101 & 24.4 & 3.70 & 0.37 & 0.74 \\
\hline
\end{tabular}

medication self-management skills measured through heiQ ${ }^{T M}$. The secondary outcome measured in this study was an improvement in lipid profile, blood pressure, heart rate, $\mathrm{BMI}$ and $\mathrm{WC}$ measurements.

The baseline demographics, disease biomarkers and lifestyle parameters trended by a simple Excel ${ }^{\mathrm{TM}}$ (Microsoft Office) data set and were then thematically analysed using IBM $^{\circledR}$ SPSS $^{\circledR}$ (version 23) and R-Studio ${ }^{\circledR}$ (version 3.2.2) software packages. The independent sample t-test was used to compare the mean values of scale variables for intervention (subgroups $A+B$ ) with non-intervention group (group $C$ ) at baseline. The one-way ANOVA test was used to compare the mean values of scale variables for the three groups $A, B$ and $C$. The chi-square test was used to compare the distribution of categorical variable values between the three groups $A, B$ and $C$.

No serious harm was observed in this study and participants with elevated HBA1c level and secondary outcomes were informed to the routine medical officers for further investigations.

\section{Results}

110 participants received the study intervention and another 56 were followed up in the usual care group. The retention rates after six months follow up were 91.8\% and $98.2 \%$ for the intervention and usual care groups respectively (Figure 1 ).

Table 1 presents an overview of baseline socio-demographic characteristics. Participant's age ranges from
27-79 years with the mean age 56.2 years (SD 8.95). A large percentage of participants were females $(72.3 \%$, 120). Educational status varied; $28.3 \%$ participants had completed General Certificate of Education Advanced Level (GCE-A/L), while $67.5 \%$ reported education up to grade 10 (GCE -Ordinary Level) or below. Considerable proportions of the sample were either unemployed $(39.1 \%)$ or self-employed (21.7\%). Almost half of the participants $(51.2 \%)$ had household earning between LKR 10,000-30,000 per month, while 37.4\% earned less than LKR 10,000. The table below illustrates the differences between males and females on several baseline characteristics, key values of gender specific medical and lifestyle factors of adults with T2DM. The median duration of time since diagnosis of diabetes ranged between 5-10 years, while the mean range lies between $2-5$ years. Overall, $75 \%$ of participants reported being diagnosed T2DM within the past 10 years, with $85 \%$ managing their diabetes with oral hypoglycaemic agents. Approximately, 1 out of 10 participants were on oral hypoglycaemic agents combined with Insulin therapy majority of them being males. The most prominent complication in both genders was peripheral neuropathy followed by eye diseases specially cataract and glaucoma.

Baseline measurements reveal that there was no significant difference between the control and intervention groups with regards to demographic, anthropometric and key biomarker variables except for diastolic pressure of the participants, which were higher in the usual care group $(P<0.05)$. 
Table 2: Diabetes complications recorded among the study participants.

\begin{tabular}{|l|l|l|l|}
\hline Diabetes Complication & Males $\mathbf{( n = 4 6 )}$ & Females $(\mathbf{n}=\mathbf{1 2 0})$ & Total $(\mathbf{n}=\mathbf{1 6 6})$ \\
\hline Eye Disease & 12 & 14 & 26 \\
\hline Kidney Disease & 03 & 02 & 05 \\
\hline Neuropathy & 20 & 42 & 62 \\
\hline Heart Disease & 04 & 01 & 05 \\
\hline Erectile Dysfunction & 02 & 0 & 02 \\
\hline
\end{tabular}

Table 3: Drugs prescribed for the treatment of type 2 diabetes at baseline.

\begin{tabular}{|l|l|l|l|}
\hline & $\begin{array}{l}\text { Intervention } \\
(\mathbf{n = 1 1 0 )}\end{array}$ & $\begin{array}{l}\text { Non-intervention } \\
(\mathbf{n = 5 6 )}\end{array}$ & $\begin{array}{l}\text { Total } \\
(\mathbf{n}=\mathbf{1 6 6})\end{array}$ \\
\hline Oral hypoglycaemic agents & 100 & 47 & 147 \\
\hline Metformin & 6 & 5 & 11 \\
\hline Tolbutamide & 18 & 12 & 30 \\
\hline Glibenclamide & 15 & 18 & 33 \\
\hline Gliclazide & 6 & 2 & 8 \\
\hline Sitagliptin & & & 21 \\
\hline Insulin treatments & 14 & 7 & 1 \\
\hline Mixtard insulin & 1 & 0 & \\
\hline Isophane insulin & 14 & \\
\hline
\end{tabular}

The differences between the intervention and usual care groups at six months follow up highlighted in Table 1. Based on primary outcome, a significant difference within and between the groups was found in $\mathrm{HbA1c}(\mathrm{p}<$ 0.001 ) after controlling the baseline values. At six months of intervention, mean $\mathrm{HbA} 1 \mathrm{c}$ level decreased from $8.6 \%$ to $7.3 \%(p<0.001)$ in the intervention group and from $8.6 \%$ to $8.2 \%(p=0.026)$ in the usual care group. A significant reduction in total cholesterol $(p<0.001)$ and LDL cholesterol $(p=0.001)$ was observed in intervention group but not in the usual care group. There was decrease in the mean values of HDL, Triglycerides in both groups, but the changes were not significant. Further statistical tests reveal that the two groups show the changes in systolic and diastolic pressure after six months, but neither of changes were significance between or among the groups. In addition, weight ( $p=0.001)$, waist circumference $(p<0.001)$ and BMI values $(p=0.002)$ were significantly reduced when compared with baseline values. However, only the weight and waist circumference provide an evidence of significant change between intervention and usual care groups.

Table 2 reveals the diabetes complications among the study participants at the initial stage of the study while Table 3 describes the common prescribing pattern of anti hyperglycaemic agents at the initiation of study.

The most prominent complication in both males (43.5\%) and females (35.0\%) was peripheral neuropathy (37.3\% of total sample) followed by eye disease (15.7\%).

\section{Discussion}

The present study was designed to determine the feasibility and efficacy of a diabetes self-management intervention based on PITS model. The approach of this study differs from other studies in local and international setups as it focused to examine the effect of health literacy on glycaemic control and the effect of culturally adapted structured education program.
Baseline characteristics of this study confirmed the findings of study conducted by Jayasuriya R, et al. in Sri Lanka, in 2015, where the majority of patients with poorer glycaemic control were females among both intervention and usual care groups [8].

The most interesting finding was that the decrease in mean $\mathrm{HbA1C}$ in the intervention group was significantly higher than that with the usual care group, providing the clear indication of superior efficacy of education intervention. The change in mean $\mathrm{HbA1c}$ level in intervention group and usual care group was $15.1 \%$ and $4.6 \%$ respectively. This study set out with the aim to achieve the glycaemic target as $7.0 \%$ for glycaemic control in the study, based on clinical guidelines for management of diabetes mellitus in Sri Lanka [19]. Prior studies that have noted the importance of reduction of $\mathrm{HbA1c}$ to $<$ $6.5 \%$ result in significant benefits in reduction of micro and macrovascular complications of T2DM $[8,20]$.

Another important facet of this study was the development of an intervention which was by an educator, provided the evidence for the feasibility of introducing diabetes educators or other professionals and their ability to influence lifestyle behaviour to be involved in health education process that reduce the work load of the physician centred clinic setting in Sri Lanka.

High retention rate of the study confirm the close professional relationship as part of the one to one follow up education, thus providing an indirect evidence of good compliance and adherence to self-care management with the special consideration of diet, exercise and adherence to the medication. This was evidence by the reduction of total cholesterol, waist circumference and weight.

The generalisability of these results is subject to certain limitations, for instance inclusion of convenience sampling and small sample size may not have been re- 
presentative of the broader population of adults with type 2 diabetes. Another limitation of this study was the exclusion of T2DM patients who were not able to communicate in Sinhala language that limits the participation of other ethnic groups to the study. The study was able to minimise any contamination between the groups, by having the two groups in different locations and visiting the facilities on separate days.

Additionally, the study participants were recruited from two large tertiary care hospitals in Sri Lanka, located in the most urban parts of the country, where people have more access to media and other information resources than those in the rural areas, which could have led to selection bias.

\section{Conclusion}

T2DM is a major public health concern in Sri Lanka. The face-to-face, one-to-one structured patient education program (based on the PITS model), which was tailored to patient's current medications, was shown to be an effective approach to diabetes care and patientcentred intervention in Sri Lanka.

The education intervention produced significant $\mathrm{HbA1c}$ control in the intervention group, found to be $7.5 \%$ for the overall intervention group and $8.2 \%$ for the non-intervention group at the end of six months period.

Although the current study is promising, it is based on small sample from two highly populated regions; a key policy priority need for a population-based study augmented with the cost effectiveness study for the long-term care of T2DM patient management that could be implemented in all national hospitals, to reverse the rising trend of diabetes in Sri Lanka.

\section{Acknowledgements}

Uditha, Bulugahapitiya, Consultant Endocrinologists, Colombo South Teaching Hospital, Kalubowila; medical officers, phlebotomists and administration staff from the Teaching Hospitals Karapitiya and Colombo South, Sri Lanka for their support during the intervention. Study participants from endocrinology clinics at Teaching hospitals Karapitiya and Colombo South, Sri Lanka for their participation.

Special thanks to Charles Darwin University, Australia and University Grant Commission Sri Lanka for providing scholarship and grants to support for the first author's study.

\section{Conflicts of Interest}

The Authors declare that there is no conflict of interest.

\section{Funding Source Declaration}

This research was supported by the University Grant Commission [grant number UGC/DRIC/PG/2015(i)/ $\mathrm{RUH} / 01,2015]$ Sri Lanka and no financial assistance for the publications.

\section{Registration of Trial}

This study was registered under the Sri Lanka Clinical Trial Registry, No: SLCTR/2015/014, which is a primary registry for clinical trials involving human subjects conducted in Sri Lanka that is linked to the Registry Network of the International Clinical Trials Registry Platform of the WHO.

\section{References}

1. L Guariguata, DR Whiting, I Hambleton, J Beagley, U Linnenkamp, et al. (2014) Global estimates of diabetes prevalence for 2013 and projections for 2035. Diabetes Res Clin Pract 103: 137-149.

2. S Wild, G Roglic, H King, A Green, R Sicree (2004) Global Prevalence of Diabetes. Diabetes Care 27: 1047-1053.

3. A Ghaffar, KS Reddy, M Singhi (2004) Burden of non-communicable diseases in South Asia. BMJ 328: 807-810.

4. International Diabetes Federation (2017) IDF Diabetes. International Diabetes Federation 6.

5. L Ounnapiruk, V Wirojratana, N Meehatchai, S Turale (2014) Effectiveness of a behaviour modification program for older people with uncontrolled type 2 diabetes. Nurs Health Sci 16: 216-223.

6. GE Caughey, Al Vitry, AL Gilbert, EE Roughead (2008) Prevalence of comorbidity of chronic diseases in Australia. BMC Public Health 8: 221-234.

7. SA Quandt, HI Edward, JK Kirk, S Saldana, SH Chen, et al. (2014) Assessment of a short diabetes knowledge instrument for older and minority adults. Diabetes Educ 40: 68-76.

8. R Jayasuriya, MJ Pinidiyapathirage, $R$ Jayawardena, A Kasturiratne, P De Zoysa, et al. (2015) Translational research for diabetes self-management in Sri Lanka: A randomized controlled trial. Prim Care Diabetes 9: 338-345.

9. AA Amarasekara, W Fongkaew, SW Wimalasekera, S Turale, Chanprasit C (2015) Cross-sectional study of glycaemic control among adults with type 2 diabetes. Nurs Health Sci 17: 223-228.

10. MH De S Jayawardena, C Idampitiya, C Jayawarna, K Wanigasuriya, GA Thomson, et al. (2007) An audit of standards of care at a Sri Lankan diabetic clinic. Diabetes Res Clin Pract 75: 249-251.

11. DP Perera, RE De Silva, WL Perera (2013) Knowledge of diabetes among type 2 diabetes patients attending a primary healthcare clinic in Sri Lanka. East Mediterr Health $\mathrm{J}$ 19: $644-648$.

12. Cooray BPR, Ball PA, Morrissey H, Waidyarathne EI (2017) The effect of Health education in the management of type 2 diabete and the the Sri Lankan perspective: A Review. Sri Lanka Journal of Diabetes Endocrinology and Metabolism 7: 34-38.

13. SL Norris, MM Engelgau, KM Narayan (2001) Effectiveness of Self-Management Training in Type 2 Diabetes: A systematic review of randomized controlled trials. Diabetes Care 24: 561-587.

14. J Wens, E Vermeire, H Hearnshaw, A Lindenmeyer, Y Biot, et al. (2008) Educational interventions aiming at improving adherence to treatment recommendations in type 2 diabetes: A sub-analysis of a systematic review of randomised controlled trials. Diabetes Res Clin Pract 79: 377-388.

15. P Katulanda, DA V Rathnapala, R Sheriff, DR Matthews (2011) Province and ethnic specific prevalence of diabetes among Sri Lankan adults. Sri Lanka Journal of Diabetes Endocrinology and Metabolism 1: 2-7. 
16. P Katulanda, GR Constantine, JG Mahesh, R Sheriff, RD Seneviratne, et al. (2008) Prevalence and projections of diabetes and pre-diabetes in adults in Sri Lanka-Sri Lanka Diabetes, Cardiovascular Study (SLDCS). Diabet Med 25: 1062-1069.

17. J Jarvis, TC Skinner, ME Carey, MJ Davies (2010) How can structured self-management patient education improve outcomes in people with type 2 diabetes? Diabetes Obes Metab 12: 12-19.

18. RH Osborne, GR Elsworth, K Whitfield (2007) The Health Education Impact Questionnaire (heiQ): An outcomes and evaluation measure for patient education and self-management interventions for people with chronic conditions. Patient Educ Couns 66: 192-201.

19. Endocrine Society of Sri Lanka (2013) Clinical Guidelines: Endocrine Management of Diabetes Mellitus. Sri Lanka Journal of Diabetes Endocrinology and Metabolism 3: 45-57.

20. UK prospective diabetes Study (UKPDS) Group (1998) Intensive blood glucose control with sulphonylureas or insulin compared with conventional treatment and risk of complications in patients with type 2 diabetes (UKPDS 33). Lancet 354: 837-853. 